\title{
Cutaneous Metastasis of Thymic Carcinoma to the Frontal Scalp
}

\author{
Leon Kou ${ }^{1}$, DO, Austin Wong, DO ${ }^{1}$, Lily Zhong, DO ${ }^{1}$, Sid Danesh, MD ${ }^{1}$ \\ 1'Danesh Dermatology, Beverly Hills, CA
}

\section{ABSTRACT}

Thymic carcinomas are a rare variant of thymic epithelial tumors. Compared to thymomas, they are much more aggressive, difficult to treat, and have a higher mortality rate. Metastasis outside of the mediastinum is rare and usually to sites such as the lung, lymph nodes, liver, pleura, or bones. We report a case of immunohistochemistry proven thymic carcinoma metastasis to the cutaneous surface of the frontal scalp in a 53-year-old Asian female.

\section{INTRODUCTION}

Thymic carcinomas (TC) are a rare and aggressive variant of thymic epithelial tumors. The symptoms of TC are often vague and caused by local invasion and mass effect. They rarely metastasize outside of the anterior mediastinum, but typically involves the lungs, lymph nodes, liver, pleura, or bones when it occurs. ${ }^{1,2}$ Skin metastases are extremely rare and could easily be misdiagnosed as benign skin conditions such as localized dermatitis or infection. TCs lack pathognomonic histology and immunohistochemistry (IHC) features. ${ }^{3}$ Ruling out differentials such as metastasis from other primary sites are often required before definitive diagnosis. ${ }^{4}$

To date, only a handful of articles have reported on cutaneous metastasis of TC and there is a scarcity of data on presentation, diagnosis, and management. ${ }^{5}$ We report a rare case of IHC confirmed cutaneous metastasis of TC.

\section{CASE REPORT}

53-year-old female presented to our dermatology clinic with an enlarging painful frontal scalp lesion for 1 year. It was previously treated as an infection with a course of antibiotics with subjective improvement to lesion size. The patient noted hair loss of the surrounding area within the past 6 months. She denied previous trauma or aggravating factors to the site. She denied all review of systems including possible symptoms of myasthenia gravis such as weakness of the ocular, respiratory, or bulbar muscles.

Her medical history was significant for TC with metastasis to the lung diagnosed 1 year ago. Her TC workup began when vague superior vena cava syndrome symptoms first presented 2 years ago. The symptoms worsened over time and a lengthy outpatient workup led to the discovery of the mediastinal mass on cardiac MRI. The patient underwent anterior mediastinotomy with surgical biopsy which revealed a diagnosis of thymic neoplasm, likely TC, with lung metastasis. July $2021 \quad$ Volume 5 Issue 4 


\section{SKIN}

She refused chemotherapy and further surgical resection and instead, underwent anterior mediastinum radiotherapy at 44 Gy/22 fractions for 1 month with some decrease in mass size on repeat CT. Previous biopsy results and imaging were unavailable for review.

The lesion appeared as an isolated erythematous subcutaneous nodule with overlying telangiectasias at the mid frontal scalp with alopecia surrounding and overlying the area of the nodule (Figure 1). Due to the patient's medical history of TC, the lesion was removed via excisional biopsy.

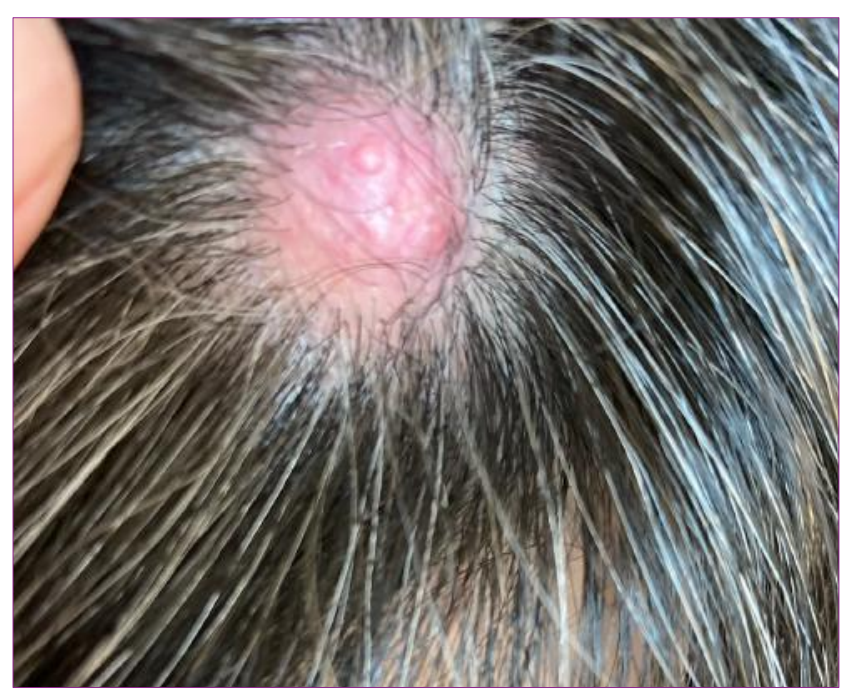

Figure 1. Erythematous subcutaneous nodule with overlying telangiectasias at the mid frontal scalp with alopecia surrounding and overlying the area of the nodule

Pathology reported multiple lobules of dermal proliferation of neoplastic epithelioid cells with nuclear pleomorphism (Figure 2). IHC staining was positive for AE1/AE3, P63, and PAX8 and negative for TTF1 and Synaptophysin (Figure 3). CD5 and CD117 staining revealed positive staining for few lymphoid cells, but negative for epithelial cells. The results were consistent with metastatic TC to the mid frontal scalp. The patient was referred to oncology to investigate the extent of metastasis to other sites.

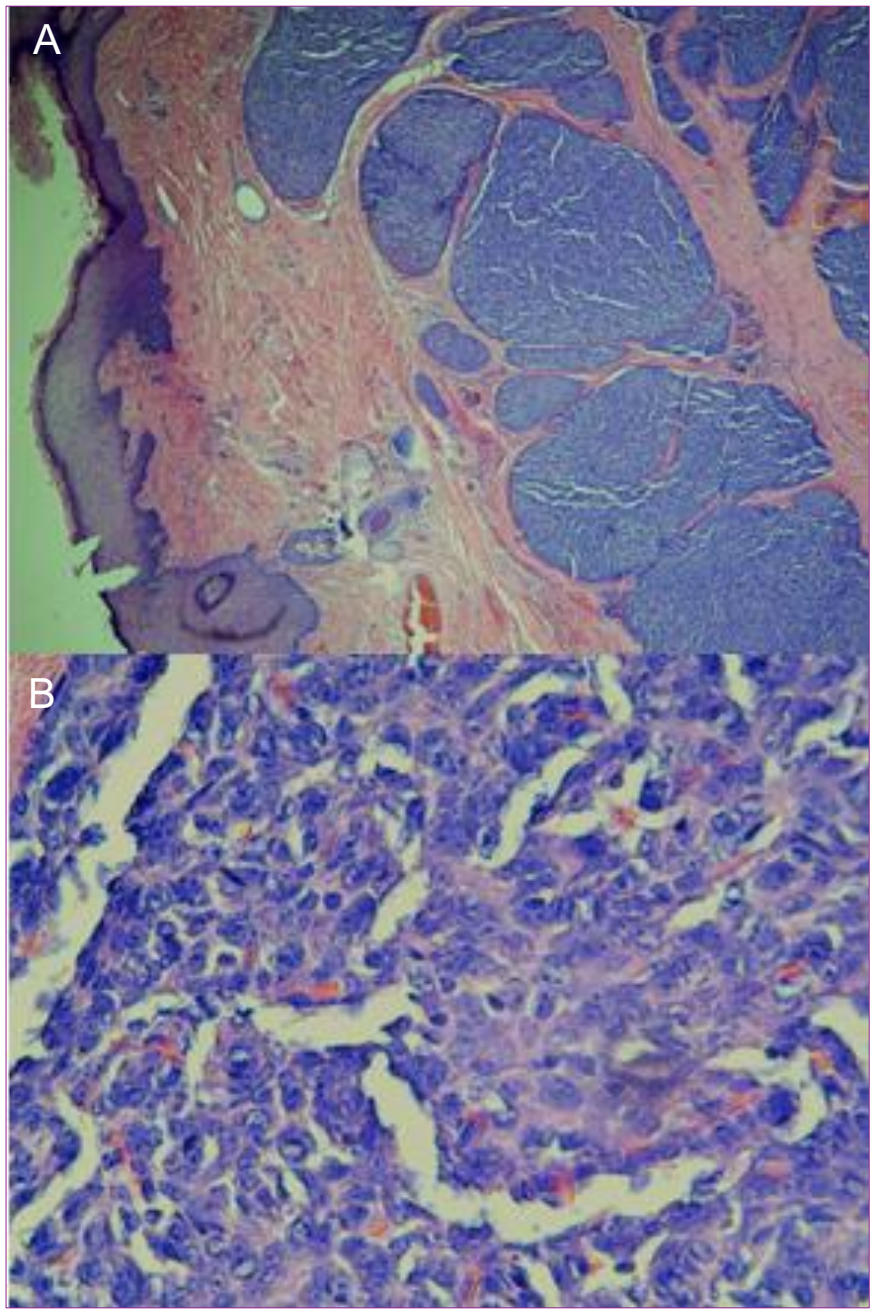

Figure 2. A) Hematoxylin and eosin (H\&E) showing multiple lobules of dermal proliferation of neoplastic epithelioid cells. Magnification x 40. B) H\&E showing tumor cells nuclear pleomorphism. Magnification $x$ 400.

\section{DISCUSSION}

Histologically, TCs usually lack features of a normal thymus and the lymphocytic components often seen in thymomas. TCs also display frank features of malignancy such as anaplasia, increased proliferative activity, and cellular atypia. ${ }^{6}$ Primary TCs have no distinguishing histological features to separate them from metastasis of other

July 2021 Volume 5 Issue 4 


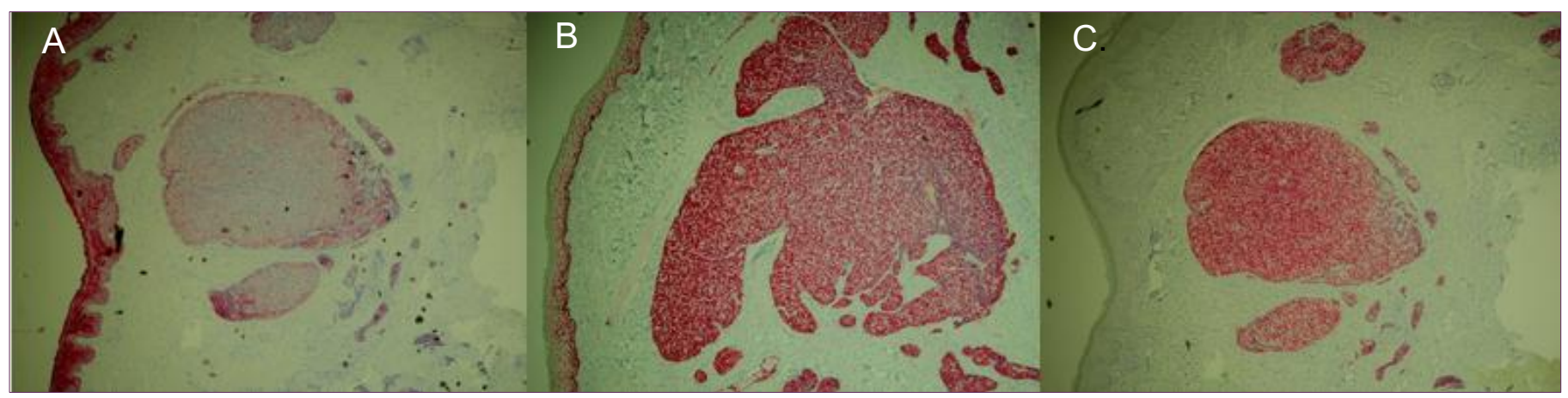

Figure 3. Positive immunohistochemistry staining of tumor epithelioid cells for A) AE1/AE3 B) P63 C) PAX8 Magnification $\times 40$.

organ systems. Notably, primary lung carcinomas must be ruled out due to proximity of presenting sites and similarity in histological appearance. ${ }^{4} \mathrm{IHC}$ plays a vital role in distinguishing TCs from metastasis from other areas into the mediastinum despite TCs lacking a distinct IHC profile. ${ }^{3}$

CD5 and CD117 are widely used markers of TC. $^{7}$ Interestingly, our sample only had positive CD5 and CD117 staining for a few lymphocytes rather than strong, diffuse cytoplasmic staining of epithelial cells as previously reported cases of extra-thoracic TC metastasis. ${ }^{1,5}$ However, lack of CD5 and CD117 positive staining does not necessarily rule out TC. In a case series of 65 TC cases, only $39 \%$ and $65 \%$ stained positive for CD5 and CD117, respectively. ${ }^{8}$ Another case series with 9 TC cases found similar results for CD5 IHC staining and hypothesized that using paraffin embedded tissue rather than frozen section for $\mathrm{IHC}$ decreased the sensitivity of positivity. $^{9} \quad$ Ultimately, investigations, with larger sample size, into the use of CD5 and CD117 for TC identification may be helpful and explain the reason behind our results.

The diagnosis of cutaneous metastasis of TC in our patient was made based on previous imaging, surgical biopsy results, and the remaining $\mathrm{IHC}$ results. Except for CD5 and
CD117, our IHC results correlate with a previously reported TC cutaneous metastasis case. ${ }^{5}$ Positive PAX8 staining, found in approximately $77 \%$ of TCs, but rarely expressed in primary lung cancers, supported the diagnosis of TC with metastasis to the lungs and our current diagnosis of TC metastasis to the skin. ${ }^{10,11}$ TCs generally stain negative for TTF- 1 and synaptophysin and our negative results further rule out the possibility of lung, thyroid, or neuroendocrine tumor metastasis. ${ }^{12}$ Positive staining for P63, found in normal thymus gland and thymic epithelial tumors, and pancytokeratin (AE1/AE3), typically positive in TCs, further support our diagnosis. ${ }^{3}$

Our patient's skin lesion appeared 1 month after discovery of the mediastinal mass by cardiac MRI and because it was left uninvestigated, the lesion worsened over 1 year with increased size, pain, and alopecia of the surrounding area. Generally, cutaneous metastasis of internal tumors is rare. They can be cosmetically devastating and are usually poor prognostic factors. Our patient is at minimum stage IIIB, with an incompletely resected TC, which puts her 5year overall survival at approximately $31 \%{ }^{4}$

July 2021 Volume 5 Issue 4 
TC cutaneous metastases are difficult to diagnose by gross appearance alone and even pathology may find it difficult without proper context. TC metastasis to sites such as the parotid and small intestine have been reported. ${ }^{1,2,5}$ Current NCCN guidelines suggest clinical correlation on using PET-CT or MRI during workup. ${ }^{4}$ More data is needed to develop an algorithmic approach to using PET-CT and MRI to rule out metastasis to other sites and organs due to the tumor's aggressiveness.

\section{CONCLUSION}

TC skin metastasis is rare and could be misdiagnosed for a benign skin condition. Careful consideration of the patient's history and clinical presentation was necessary to have TC metastasis as a differential. Without using immunohistochemistry stains, diagnosis and ruling out metastasis from other primary cancers would have been difficult if not impossible.

\section{Conflict of Interest Disclosures: None}

Funding: None

\section{Corresponding Author:}

Leon Kou, DO

240 S. La Cienega Blvd

Beverly Hills, CA 90211

Phone: 909-859-9885

Email: k.leon@ucla.edu

\section{References:}

1. Huang P, Huang R, Xiao W, et al. Ectopic thymic carcinoma in parotid gland: a rare case and review of literature. Int J Clin Exp Pathol. 2019;12(1):384-388.

2. Yuan $Y$, Pu H, Pang M, Liu Y, Li H. Thymic carcinoma metastasize to the small intestine: a case report. BMC Gastroenterol. 2020;20. doi:10.1186/s12876-020-01505-7

3. Weissferdt A, Moran CA. Immunohistochemistry in the diagnosis of thymic epithelial neoplasms. Appl Immunohistochem Mol Morphol.
2014;22(7):479-487. doi:10.1097/PAl.0b013e3182a53856

4. National Comprehensive Cancer Network (NCCN). Clinical Practice Guidelines in Oncology: Thymomas and Thymic Carcinomas. Version 1.2021. Accessed at https://www.nccn.org/professionals/physician_gls/ pdf/thymic.pdf on April 21, 2021.

5. Choi SJ, Rho NK, Yang JM, Lee ES. A Case of Cutaneous Matestasis Originating from Thymic Carcinoma. Annals of Dermatology. 2016;14(4):230-234.

6. Eng TY, Fuller CD, Jagirdar J, Bains $Y$, Thomas CR. Thymic carcinoma: state of the art review. Int $J$ Radiat Oncol Biol Phys. 2004;59(3):654-664. doi:10.1016/j.jirobp.2003.11.021

7. Kriegsmann M, Muley T, Harms A, et al. Differential diagnostic value of CD5 and CD117 expression in thoracic tumors: A large scale study of 1465 non-small cell lung cancer cases. Diagn Pathol. 2015;10. doi:10.1186/s13000-015-0441-7

8. Weissferdt A, Moran CA. Thymic carcinoma, part 1: a clinicopathologic and immunohistochemical study of 65 cases. Am J Clin Pathol. 2012;138(1):103-114. doi:10.1309/AJCP88FZTWANLRCB

9. Berezowski K, Grimes MM, Gal A, Kornstein MJ. CD5 immunoreactivity of epithelial cells in thymic carcinoma and CASTLE using paraffinembedded tissue. Am J Clin Pathol. 1996;106(4):483-486. doi:10.1093/ajcp/106.4.483

10. Jeong JH, Kim NY, Pyo J-S. Analysis of PAX8 immunohistochemistry in lung cancers: a metaanalysis. J Pathol Trans/ Med. 2020;54(4):300309. doi:10.4132/jptm.2020.06.08

11. Weissferdt A, Moran CA. Pax8 expression in thymic epithelial neoplasms: an immunohistochemical analysis. Am J Surg Pathol. 2011;35(9):1305-1310. doi:10.1097/PAS.0b013e3182260735

12. Zamecnik J, Kodet R. Value of thyroid transcription factor-1 and surfactant apoprotein A in the differential diagnosis of pulmonary carcinomas: a study of 109 cases. Virchows Arch. 2002;440(4):353-361. doi:10.1007/s00428$001-0552-2$ 